\title{
UMA ABORDAGEM MULTICRITÉRIOS PARA RECAPACITAÇÃO DE LINHAS DE TRANSMISSÃO
}

\author{
Ricardo S. Cavassin* \\ rcavassin@hotmail.com
}

\author{
Thelma S. Piazza Fernandes* \\ thelma@eletrica.ufpr.br
}

${ }^{*}$ Centro Politécnico - DELT-TC-UFPR

Caixa Postal - 19011

CEP 81531-980 - Curitiba PR

\section{ABSTRACT}

\section{A Multicriteria Approach to Retrofit of Transmission Lines}

The crescent demand for electricity overloads the transmission system which requires constant increases to not be a hindrance to development. However, the construction of new transmission lines has faced many obstacles due to the difficulty to constitute new intervention area and the need to reduce environmental impacts. A very advantageous solution to these problems is the retrofit of the transmission lines, which can be made in many ways. One of the most common is the replacement of power cables, which manufacturers continually invest and create new materials to allow new options of design. Moreover, just retrofit lines already present problems of ampacity's superation, justifying the development of a multi-criteria methodology that adequately ponder the objectives during the choice of an adequate handle. The criteria used in this work are the maximization of height handle- round, ampacity and supervened of the line (in function of the exceeding ampacity) and, minimization of implantation's cost, losses and addition of efforts at the structures. The optimization problem involved is resolved by the Weighed Global Criterion Method. The results of the methodology (that effects the calculations of parameters of the available handles and selects to better to be installed) are presented for one line transmission of $230 \mathrm{kV}$ installed at the state of Paraná.

Artigo submetido em 25/04/2011 (Id.: 1327)

Revisado em 17/09/2011, 16/04/2012

Aceito sob recomendação do Editor Associado Prof. Eduardo N. Asada
KEYWORDS: Retrofit, Multiobjective Optimization, Transmission Line

\section{RESUMO}

A crescente demanda de energia elétrica sobrecarrega o sistema de transmissão que requer constantes ampliações para não prejudicar o desenvolvimento. Porém a construção de novas instalações de transmissão vem enfrentando diversos obstáculos em função da dificuldade de constituir novas faixas de servidão e pela necessidade de redução dos impactos ambientais. Uma solução que se apresenta muito vantajosa a estes problemas é a recapacitação da linha de transmissão. Dentre os vários métodos existentes para tal, destacase a substituição dos cabos condutores, cujos fabricantes têm investido constantemente na pesquisa de novos materiais e ligas. Além disto, observa-se que em alguns casos linhas recém recapacitadas já apresentam problemas de superação de ampacidade, exigindo-se assim, o desenvolvimento de uma metodologia multi- ritérios que pondere adequadamente os objetivos envolvidos no processo de escolha de um cabo. Os critérios utilizados neste trabalho são maximização de altura cabo-solo, maximização da ampacidade, maximização da sobrevida da linha em função da ampacidade excedente, minimização dos custos de implantação, perdas e acréscimo de esforços nas estruturas. O problema de otimização envolvido é resolvido pelo Método do Critério Global Ponderado. Os resultados da metodologia (que efetua os cálculos dos parâmetros dos cabos disponíveis e seleciona o melhor cabo a ser instalado) são apresentados para uma linha de transmissão de 230 kV instaladas no estado do Paraná. 
PALAVRAS-CHAVE: Recapacitação, Otimização Multiobjetivo, Linhas de Transmissão.

\section{INTRODUÇÃO}

O constante aumento da demanda de energia elétrica faz com que haja necessidade de ampliação dos sistemas de transmissão, como subestações e linhas de transmissão, impedindo assim que instalações precárias sejam fatores limitantes do desenvolvimento econômico.

A implantação de novas linhas de transmissão (LT) vem enfrentando dificuldades em função de alguns fatores externos que tem impacto direto no custo dessas linhas. Primeiramente a minimização do impacto ambiental, que provoca a utilização de estruturas mais altas e consequentemente mais pesadas e também faz com que o traçado a ser seguido não seja necessariamente o mais curto. Outro problema é o alto custo para se constituir novas faixas de servidão de passagem e para o pagamento de indenizações.

Tendo em vista essas dificuldades, torna-se vantajosa a recapacitação das linhas de transmissão, que consiste no aumento da capacidade de transmissão de energia elétrica de uma instalação existente com o mínimo impacto possível.

Vários são os métodos utilizados para a recapacitação de uma linha de transmissão, sendo que os mais comuns são o retensionamento dos cabos condutores, alteração de padrões construtivos, substituição dos condutores e a alteração do nível de tensão, que é utilizado em menor escala (Oliveira, 2000).

De modo geral, a literatura apresenta estudos de recapacitação que utilizam cabos especiais. Nesses estudos, testamse um ou dois tipos de cabos comparando-os com o existente e escolhendo-se pelo que apresenta melhor desempenho (Dutra et al., 2005; Hoffmann and Fonseca, 2003; Stephan and Costa, 2008; Wiedmer et al., 2007).

Em geral a escolha do cabo mais apropriado para recapacitar linhas de transmissão é realizada com base na experiência dos profissionais da área, pois apesar de existir uma pequena quantidade de opções de cabos, existe por outro lado, a dificuldade de se testar todas essas opções a fim de se escolher o melhor cabo condutor a ser utilizado. Além disto, segundo depoimentos destes profissionais da área, é freqüente a superação dos limites de capacidade da linha tão logo a mesma seja recapacitada.

Assim, as dificuldades e empirismos encontrados nessa área motivaram o desenvolvimento de uma metodologia multicritérios que permite o automatismo e otimização do processo de escolha do melhor cabo a ser utilizado em um projeto de recapacitação. Essa metodologia pondera as grandezas físicas envolvidas no processo de escolha do cabo que são altura cabo-solo da linha, sobrevida da linha em função da ampacidade excedente, custos de implantação, perdas e esforços nas estruturas.

A disposição deste trabalho é dada como se segue: inicialmente apresenta-se o problema de recapacitação de linhas de transmissão. Na seqüência, é apresentada a formulação matemática do problema e, finalmente, é feita a apresentação de resultados e a conclusão.

\section{PROJETOS ELETROMECÂNICOS DE LINHAS DE TRANSMISSÃO}

O projeto eletromecânico propriamente dito de uma LT se inicia após a escolha de um traçado viável entre os dois pontos que se deseja interligar, definição da classe de tensão, tipos de estruturas, bitola e composição dos condutores e outros.

Já o projeto mecânico de uma LT, consiste na determinação dos esforços atuantes sobre os elementos que a compõe.

Segundo Labegalini et al. (1992), os trabalhos de projeto mecânico das LTs se dividem em:

- Projetos dos cabos e dos suportes sobre os perfis dos terrenos;

- Projetos dos suportes;

- Projetos das fundações.

A escolha dos tipos e bitolas dos cabos condutores obedece a critérios econômicos e técnicos e muito raramente a critérios mecânicos. A escolha da série e tipos de estruturas a serem utilizadas, suas dimensões e sua configuração obedecem aos critérios mecânicos e elétricos, o terreno onde serão implantadas, bem como considerações de segurança.

Para se determinar a altura e disposição dos suportes, utilizase como parâmetro o cálculo da mínima altura cabo solo e para isto é determinada a flecha que o cabo descreve quando apoiado em dois suportes.

A Figura 1 apresenta um cabo estendido entre dois suportes de alturas diferentes, cuja diferença é dada por h, e com um vão horizontal igual a A (vão).

A flecha $f s$ representa a maior distância vertical entre a linha que liga os pontos de apoio do cabo e um ponto da curva e pode ser determinada da seguinte forma: 
Figura 1: Cabos suspensos entre suportes com alturas diferentes (Labegalini et al., 1992).

$$
f_{s}=\frac{T_{0}}{p} \cdot\left[\cosh \left(\frac{A}{2 \cdot \frac{T_{0}}{p}}\right)-1\right]
$$

onde:

$T_{0}$ : tração com direção tangente à curva [kgf];

$f: \quad$ flecha pela equação da catenária $[\mathrm{m}]$;

$p$ : peso unitário do condutor $[\mathrm{kgf} / \mathrm{m}]$;

$A$ : vão [m].

Para cada estrutura é feito o cálculo dos esforços longitudinais, verticais e transversais, de acordo com as características dos cabos.

Os esforços verticais são consequência do peso próprio do cabo e do vão gravante da estrutura (vão gravante é a distância entre os vértices das catenárias dos vãos adjacentes à estrutura).

Portanto, conclui-se como parâmetros importantes dos cabos na determinação dos esforços nas estruturas o seu peso próprio, sua tração de ruptura e o seu diâmetro.

\section{RECAPACITAÇÃO DE LINHAS DE TRANSMISSÃO}

Recapacitar uma linha de transmissão consiste em aumentar sua capacidade de transmissão de energia elétrica para atender a uma nova solicitação do sistema em situações nas quais seja inviável a implantação de uma nova LT. Ou seja, consiste em aumentar seu limite térmico ou a sua temperatura máxima de operação, chamada de temperatura de projeto.

Para tanto, é necessário calcular qual será o limite de corrente permitido a circular na linha de transmissão para que o cabo não ultrapasse a temperatura de projeto. A determinação dessa corrente é feita através do cálculo da ampacidade.
A ampacidade está relacionada à capacidade de transmissão de energia elétrica de uma determinada linha de transmissão, calculada no vão crítico, que tem maior possibilidade de violação da altura cabo solo quando a LT opera na condição nominal (Oliveira, 2000).

Há vários métodos para o cálculo da ampacidade e para que ele seja feito são usados valores conservativos sugeridos pela norma NBR 5422/85 (ABNT, 1985), conforme segue:

- Radiação solar de $1000 \mathrm{~W} / \mathrm{m}^{2}$;

- Velocidade do vento $\leq 1 \mathrm{~m} / \mathrm{s}$;

- Temperatura média máxima do ar.

Um método bastante utilizado é o método simplificado para a elaboração de curvas de ampacidade (descrito a seguir), o qual é aceitável para a maioria das aplicações práticas (Labegalini et al., 1992).

Um cabo atinge a temperatura em regime permanente quando houver equilíbrio entre o calor ganho e o calor perdido pelo cabo. O cabo ganha calor principalmente por efeito Joule e pela radiação solar.

O ganho de calor por efeito Joule é consequência da circulação de corrente pelo condutor:

$$
q_{j}=I^{2} \cdot r
$$

onde:

$q_{j}: \quad$ ganho de calor por efeito Joule [W/km];

$I$ : $\quad$ corrente que circula pelo condutor $[\mathrm{A}]$;

$r: \quad$ resistência do condutor à temperatura de equilíbrio $[\Omega / \mathrm{km}]$.

No cálculo do ganho de calor por radiação solar, é considerado o valor médio indicativo em climas temperados:

$$
q_{s}=204 . d
$$

onde:

$q_{s}:$ do ganho de calor por radiação solar $[\mathrm{W} / \mathrm{m}]$;

$d$ : diâmetro nominal do cabo [m].

A perda de calor do cabo ocorre por irradiação e por convecção. A perda de calor por convecção é dada por: 


$$
\begin{aligned}
& q_{c}=945,6 \cdot\left(t-t_{0}\right) \cdot 10^{-4} . \\
& \quad \cdot\left[0,32+0,43 \cdot(45946,8 \cdot d \cdot V)^{0,52}\right]
\end{aligned}
$$

onde:

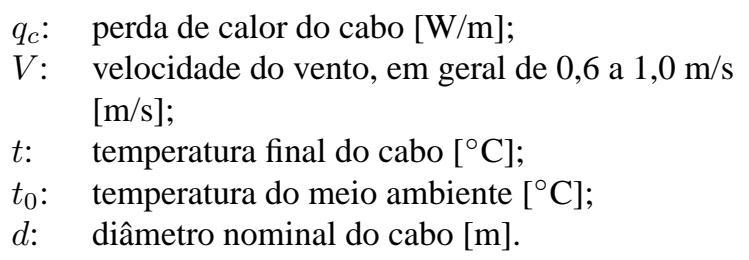

A perda de calor por irradiação é determinada da seguinte forma:

$$
q_{r}=179,2 \cdot 10^{3} . \varepsilon . d .\left[\left(\frac{T}{1000}\right)^{4}-\left(\frac{T_{0}}{1000}\right)^{4}\right]
$$

onde:

$$
\begin{aligned}
& q_{r}: \quad \text { perda de calor por irradiação }[\mathrm{W} / \mathrm{m}] \text {; } \\
& \varepsilon \text { : emissividade - varia de } 0,23 \text { a } 0,90 \text {, conforme a cor } \\
& \text { do cabo. Para cabos de alumínio, é recomendado } \epsilon \\
& \approx 0,5 \text {; } \\
& d \text { : diâmetro nominal do cabo [m]; } \\
& T: \quad \text { temperatura absoluta final do cabo }(273+\mathrm{t})[\mathrm{K}] \text {; } \\
& T_{0} \text { : temperatura absoluta do ambiente }(273+\mathrm{t} 0)[\mathrm{K}] \text {. }
\end{aligned}
$$

Desta forma a equação do equilíbrio é:

$$
q_{j}+q_{s}=q_{r}+q_{c}
$$

Substituindo (2) em (6) obtém-se o valor da ampacidade:

$$
I=\sqrt{\frac{\left(q_{r}+q_{c}-q_{s}\right) \cdot 10^{3}}{r}}
$$

onde:

$$
\text { I: ampacidade [A]. }
$$

O valor de ampacidade representa o valor máximo de corrente que deverá circular pelo cabo condutor para que ele atinja a temperatura para a qual foi projetado, mantendo assim os valores de flecha calculados.

\subsection{Tecnologias de Condutores}

O estudo de novas tecnologias de cabos condutores vem da necessidade de se atingir valores térmicos cada vez maiores com menores valores de dilatação. Os fabricantes procuram, com a utilização de novos materiais tanto no condutor como na alma de aço, desenvolver cabos com bom diâmetro de condutor, peso unitário ou tração de ruptura, ampacidade, coeficiente de dilatação linear e temperatura de operação.

A alguns cabos mais usuais são:

- Tipo ACSR: mais utilizado em LTs, também chamados de CAA (alumínio com alma de aço) e compostos por fios de alumínio 1350 e de aço galvanizado concentricamente enrolados. O núcleo é feito de aço galvanizado e a camada externa é de alumínio.

Diferentes combinações de aço-alumínio permitem obter altas cargas de ruptura sem prejuízo à ampacidade. Os fios de aço são galvanizados, ou como alternativa podem ser tratados com camadas de alumínio (ACSR AZ e ACSR AW).

- Tipo ACSR TW: são semelhantes aos cabos ACSR, porém os fios de alumínio têm forma trapezoidal, preenchendo alguns espaços que se formam nos fios circulares. Os cabos ACSR TW são relacionados de duas formas com os cabos ACSR: podem possuir igual área de alumínio e menor diâmetro, provocando uma economia de estruturas, ou igual diâmetro e maior ampacidade que o ACSR equivalente.

- Tipo ACAR: são semelhantes aos cabos ACSR, porém os fios centrais são de alumínio liga 6201, que oferecem maior ampacidade, porém menor carga de ruptura e menor peso que o cabo CAA de mesmo diâmetro.

- Tipo AAAC: todos os fios são em alumínio liga 6201, permitindo uma boa relação carga de ruptura/peso unitário, obtendo-se menores flechas que o cabo CAA de mesmo diâmetro.

- Tipo ACSS: são cabos projetados para operar continuamente em elevadas temperaturas, podendo chegar a 200 ${ }^{\circ} \mathrm{C}$ sem que ocorra perda nas características mecânicas. É formado por fios de aço centrais com camadas de alumínio liga 1350 sobrepostas. Devido às características da liga, o aço suporta praticamente toda a resistência mecânica. Tem flechas menores que os cabos CAA e, portanto apresenta vantagens na recapacitação de linhas de transmissão e em projetos de linhas que operem em altas temperaturas. No entanto, o aumento da ampacidade provoca um aumento nas perdas por aquecimento. 


\subsection{Levantamento de Dados}

Existem vários métodos para aumentar a capacidade de uma LT e todos partem de uma instalação existente, da qual é necessário o levantamento de algumas informações sobre a situação atual, que servirão de dados para o novo projeto.

O primeiro passo para iniciar o projeto é o levantamento topográfico de toda a instalação existente.

Neste levantamento é feita uma revisão geral do traçado e da LT, pontos de instalação das estruturas, ângulos, altura cabosolo e ponto de fixação dos cabos nas cadeias em todos os vãos, vegetação e outros.

\subsection{Métodos de Recapacitação de LT}

O aumento da ampacidade da linha através do aumento do seu limite térmico provoca um aumento nos valores das flechas dos cabos condutores e consequentemente a diminuição da altura cabo solo. Os métodos de recapacitação procuram evitar que esta altura atinja valores menores que os mínimos aceitáveis segundo a norma NBR 5422/85 (ABNT, 1985). Esta distância de segurança pode ser calculada da seguinte forma:

$$
\begin{aligned}
\text { Se } D_{U} \geq 87 k V & \\
\qquad D & =a+0,01 \cdot\left(\frac{D_{U}}{\sqrt{3}}-50\right) \\
\text { e se } D_{U} \leq 87 k V & \\
D & =a
\end{aligned}
$$

onde:

$$
\begin{array}{ll}
D: & \text { distância de segurança; } \\
a: & \text { distância básica, obtida pela Tabela 1; } \\
D_{U}: & \text { delta tensão de operação da linha. }
\end{array}
$$

A Tabela 1 apresenta os valores de a para o cálculo da distância mínima a ser considerada entre o cabo mais baixo da linha de transmissão em sua temperatura de projeto e os vários tipos de obstáculos encontrados.

No cálculo da distância dos condutores a superfícies de águas navegáveis, o valor de $\mathrm{H}$ corresponde à altura em metros do maior mastro e deve ser fixado pela autoridade responsável pela navegação na via considerada.

\subsubsection{Recapacitação de LT com Acréscimo da Tra- ção dos Cabos Condutores}

O primeiro método e o mais simples de recapacitação consiste no aumento da tração dos cabos condutores e a regu-
Tabela 1: Distâncias básicas (NBR 5422).

\begin{tabular}{|l|c|}
\hline $\begin{array}{l}\text { Natureza da região ou obstáculo } \\
\text { atravessado pela linha ou que dela se } \\
\text { aproxime }\end{array}$ & $\begin{array}{c}\text { Distância } \\
\text { básica a } \\
(\mathrm{m})\end{array}$ \\
\hline Locais acessíveis apenas a pedestres & 6,0 \\
\hline Locais onde circulam máquinas agrícolas & 6,5 \\
\hline Rodovias, ruas e avenidas & 8,0 \\
\hline Ferrovias não eletrificadas & 9,0 \\
\hline $\begin{array}{l}\text { Ferrovias eletrificadas ou com previsão de } \\
\text { eletrificação }\end{array}$ & 12,0 \\
\hline Suporte de linha pertencente à rodovia & 4,0 \\
\hline Águas navegáveis & $\mathrm{H}+2,0$ \\
\hline Águas não navegáveis & 6,0 \\
\hline Linhas de energia elétrica & 1,2 \\
\hline Linhas de telecomunicações & 1,8 \\
\hline Telhados e terraços & 4,0 \\
\hline Paredes & 3,0 \\
\hline Instalações transportadoras & 3,0 \\
\hline Veículos ferroviários e rodoviários & 3,0 \\
\hline
\end{tabular}

larização do padrão construtivo. Com os dados obtidos no levantamento topográfico, é possível determinar qual a tração dos cabos condutores na situação de operação e também na condição de maior duração.

Sem a utilização de dispositivos amortecedores, os cabos CAA podem ser submetidos a uma tração de até $20 \%$ de sua tração de ruptura na temperatura EDS (condição de maior duração), e este método consiste em elevar a tração do cabo condutor até este limite, diminuindo assim a flecha e aumentando a altura cabo-solo.

Dentre os métodos de recapacitação, é o de mais fácil implementação, pois permite que a linha de transmissão seja religada em um curto espaço de tempo caso seja necessário. Seus custos são reduzidos, mas se houver a necessidade de implantação de muitas estruturas, passa a ser inviável.

\subsubsection{Recapacitação de LT com substituição dos Cabos Condutores}

Na impossibilidade da execução da recapacitação com retensionamento dos cabos condutores, parte-se para a substituição dos condutores.

Para que seja feita a substituição dos condutores, é necessário obter a informação sobre qual é o tipo do condutor utilizado no projeto das estruturas para determinação do seu vão gra- 
vante e vão médio. Procura-se utilizar primeiramente cabos que tenham características mecânicas iguais ou menores do que o cabo originalmente projetado. As características a serem consideradas são:

- Peso unitário do cabo - tem influência na força vertical a ser suportada pela estrutura metálica;

- Tração máxima de ruptura - tem influência na força longitudinal, principalmente nas estruturas em ângulo e fim de linha;

- Diâmetro do cabo - tem influência na força transversal que a estrutura deve suportar.

As considerações a respeito das características relacionadas acima permite um novo projeto com aumento de capacidade da linha de transmissão sem que haja necessidade de substituição de estruturas devido ao aumento de carga mecânica nas mesmas.

Se ainda assim não houver possibilidade da obtenção dos valores de ampacidade desejados, faz-se necessário a substituição dos cabos condutores por cabos com características mecânicas maiores do que o cabo instalado. Este tipo de projeto requer uma análise minuciosa das estruturas metálicas e fundações. Em geral é necessária a substituição e reforço de algumas estruturas e o reforço de fundações.

Existem ainda outros métodos de recapacitação, cujos impactos em projetos e execução são maiores, por exemplo:

- Lançamento de mais um subcondutor por fase ou expansão do feixe de cabos condutores - estes métodos exigem que a linha opere com feixe de cabos ou que as estruturas e arranjos de cadeias de isoladores da linha de transmissão permitam a utilização de feixes;

- Mudança na tensão operativa da linha - neste caso, além da linha de transmissão, há a necessidade de uma detalhada análise nas subestações envolvidas e a reação no sistema interligado.

Como a substituição dos cabos condutores é uma prática muito usual de recapacitação por proporcionar um aumento significativo na ampacidade da linha, essa foi a escolhida nesse artigo.

\section{FORMULAÇÃO DO PROBLEMA DE OTI- MIZAÇÃO}

Existem várias tecnologias de cabos disponíveis para serem utilizados em um projeto de recapacitação. Mas, atualmente, com as ferramentas existentes, não é possível testar todas as possibilidades a fim de verificar qual é a melhor para uma determinada linha em estudo. Normalmente, escolhem- se, pela experiência do projetista, apenas alguns cabos que então são simulados e analisados.

O que se propõe neste artigo é a apresentação de uma metodologia onde a partir do levantamento topográfico da linha em estudo, calculam-se automaticamente todos os parâmetros essenciais (seção 2) para todos os cabos disponíveis. A seguir, a partir da formulação de um problema de otimização multi-objetivo, selecionam-se os cabos mais adequados.

Como esse problema de escolha de cabos para recapacitação tem um campo e busca pequeno, ou seja, o número de cabos disponíveis no mercado gira em torno de apenas 20 tipos de cabos separados em torno de 5 tecnologias, é desnecessário utilizar qualquer técnica de inteligência artificial, sendo escolhido para resolução deste problema uma técnica tradicional tal como proposta por Coello (1999) que é o Método do Critério Global Ponderado.

Este método consiste na aplicação de um peso aos critérios da função objetivo. Na prática, o método é útil na geração de subconjuntos de Pareto caracterizados pelas preferências impostas pelos pesos utilizados em cada objetivo. Matematicamente o método é expresso por:

$$
F O=\sum_{i=1}^{n} w_{i} \cdot f_{i}
$$

onde $w_{i}$ são pesos a serem usados para dar mais relevância a uma função.

A função multiobjetivo $(F 0)$ a ser minimizada para se escolher o cabo mais adequado para a recapacitação é:

$$
F O\left[f_{1}, f_{2}, f_{3}, f_{4}, f_{5}, f_{6}\right]
$$

onde:

$f_{1}$ : maximização de altura cabo-solo;

$f_{2}$ : maximização da ampacidade;

$f_{3}$ : maximização da possibilidade de expansão em função da ampacidade excedente;

$f_{4}:$ minimização de custo;

$f_{5}$ : minimização de perdas;

$f_{6}$ : minimização de acréscimo de esforços nas estruturas.

Esses critérios são tratados adequadamente no sentido de torná-las adimensionais (devido às diferentes unidades de 
cada função) e de homogeneizá-las, isto é, transformandoas em funções de minimização.

\subsection{Maximização de altura cabo solo}

Para cada cabo, deve-se calcular o valor da altura cabo solo $\left(h_{\text {cabosolo }}\right)$ para cada vão, $i$, e verificar se ocorre violação em relação ao valor mínimo determinado pela NBR 5422/85 (ABNT, 1985) (Tabela 1).

O desvio entre o valor da altura mínima e a altura cabo solo para cada vão, $i$, é definido como:

$$
\text { desvio }_{i}=h_{\text {cabosolo }}-\text { alturaminima } i=1, \ldots, n v
$$

onde $n v$ : número de vãos da linha em estudo.

Não é admissível que a altura mínima seja violada, ou seja, quando para um determinado cabo, o valor calculado da altura cabo solo $\left(h_{\text {cabosolo }}\right)$ for menor do que o valor mínimo (alturaminima), descarta-se esse cabo.

A Tabela 2 apresenta as regras de comparação para obtenção do valor do desvio.

Tabela 2: Regras para obtenção das violações de altura cabo solo.

\begin{tabular}{|c|c|}
\hline Se & Então \\
\hline$h_{\text {cabosolo }}<$ alturaminima & Cabo descartado \\
\hline$h_{\text {cabosolo }}>$ alturaminima & $\begin{array}{c}\text { desvio }_{i}=h_{\text {cabosolo }}- \\
\text { alturaminima }\end{array}$ \\
\hline
\end{tabular}

O desvio total da linha em estudo, desviocabo, é igual à soma dos desvios calculados para cada vão:

$$
\text { desviocabo }=\sum_{i=1}^{n v} \text { desvio }_{i}
$$

A fim de normalizar esse critério e transformá-lo em uma função de minimização, adota-se como função, $f_{1}$, a relação entre o desvio do cabo atual pelo desvio do novo cabo:

$$
f_{1}=\frac{\text { desviocabo }_{\text {atual }}}{\text { desviocabo }}
$$

onde:

$$
\begin{array}{ll}
\text { desviocabo }_{\text {atual }}: & \begin{array}{l}
\text { desvio calculado para o cabo } \\
\text { instalado na linha; }
\end{array} \\
\text { desviocabo novo: } & \begin{array}{l}
\text { desvio calculado para o cabo que está } \\
\text { sendo simulado. }
\end{array}
\end{array}
$$

Os passos que devem ser calculados para a determinação da altura cabo solo para cada cabo são (Labegalini et al., 1992):

\section{Passo a: Determinação da Condição Regente}

A linha de transmissão é dividida em tramos, que consiste no trecho de linha entre duas estruturas de ancoragem. O primeiro passo é definir a condição regente que é determinar entre as temperaturas de EDS, mínima e coincidente qual será a determinante para o projeto do tramo. Em seguida é utilizada a equação de estados para, sabendo a condição regente, determinar qual será a tração do cabo para a temperatura de projeto.

$$
\begin{array}{r}
T_{02}^{3}+T_{02}^{2} \cdot\left[\frac{e \cdot s \cdot p^{2} \cdot A}{24 \cdot T_{01}^{2}}+e . s \cdot \alpha \cdot\left(t_{2}-t_{1}\right)-T_{01}\right]- \\
-\frac{e \cdot s \cdot p^{2} \cdot A^{2}}{24}=0
\end{array}
$$

onde:

$T_{02}$ : valor da tração na temperatura $\mathrm{t}_{2}[\mathrm{kgf}]$;

$T_{01}$ : valor da tração na temperatura $\mathrm{t}_{1}[\mathrm{kgf}]$;

$e: \quad$ módulo de elasticidade do condutor $\left[\mathrm{kgf} / \mathrm{mm}^{2}\right]$;

$s: \quad$ área de seção transversal do condutor $\left[\mathrm{mm}^{2}\right]$;

$p$ : $\quad$ peso unitário do condutor $[\mathrm{kgf} / \mathrm{m}]$;

$A$ : comprimento do vão (vão isolado) ou valor do vão regulador (vão contínuo)[m];

$\alpha: \quad$ coeficiente de dilatação térmica linear do condutor $\left[1 /{ }^{\circ} \mathrm{C}\right]$;

$t_{1}$ : temperatura cuja tração é conhecida $\left[{ }^{\circ} \mathrm{C}\right]$;

$t_{2}$ : temperatura cuja tração se deseja calcular $\left[{ }^{\circ} \mathrm{C}\right]$.

\section{Passo b: Cálculo da Flecha}

Para a determinação da flecha do condutor na temperatura de projeto, é necessário o valor da tração do condutor nesta condição $\left(T_{0}\right)$, obtida no item anterior. Com os dados do peso unitário do cabo (p) e do comprimento do vão (A), calcula-se a flecha da seguinte forma:

Considerando: $C_{1}=\frac{T_{0}}{p}$

$$
f=\frac{T_{0}}{p} \cdot\left(\cosh \left(\frac{A}{2 *\left(C_{1}\right)}\right)-1\right)
$$


onde:

$T_{0}: \quad$ tração com direção tangente à curva [kgf];

$f: \quad$ flecha pela equação da catenária $[\mathrm{m}]$;

$p$ : $\quad$ peso unitário do condutor $[\mathrm{kgf} / \mathrm{m}]$;

$A$ : vão [m].

\section{Passo c: Cálculo da Flecha e Altura Cabo-solo no Ponto Crítico}

A partir dos dados da LT, sabe-se o ponto crítico em cada vão. Por ser o ponto no qual o cabo apresenta a menor altura cabo-solo, é necessário o cálculo da flecha neste ponto, pois com ela é possível calcular a altura cabo-solo para os diversos cabos.

O valor da altura cabo-solo serve de parâmetro de comparação entre os cabos.

Para a determinação da altura cabo solo soma-se o valor da flecha ao valor da altura de fixação do cabo na estrutura anterior $\left(h_{a}\right)$, considerando a altitude e subtrai-se o valor da altitude do ponto de violação $\left(h_{\text {violao }}\right)$ :

$$
h_{\text {cabosolo }}=\left(f_{\text {crtico }}+h_{a}\right)-h_{\text {violao }}
$$

O valor encontrado da altura cabo-solo para cada vão é comparado com o valor mínimo da distância de segurança calculada pelas equações (18) e (19), de acordo com a norma NBR 5422/85 (ABNT, 1985) e conforme Tabela 1 (distância básica - a).

$$
\begin{aligned}
\text { Se } D_{U} \geq 87 k V & \\
D & =a+0,01 .\left(\frac{D_{U}}{\sqrt{3}}-50\right) \\
\text { e se } D_{U} \leq 87 k V & \\
D & =A
\end{aligned}
$$

onde:

$D$ : distância de segurança;

$a$ : $\quad$ distância básica, obtida pela Tabela 1;

$D_{U}:$ delta tensão de operação da linha.

\subsection{Maximização de ampacidade}

O valor da ampacidade é calculado para os cabos não descartados do item anterior $\left(I_{\text {calculado }}\right)$ e para o cabo atual instalado na linha $\left(I_{\text {atual }}\right)$ conforme seção 3 .
A fim de normalizar o valor desse critério e transformá-lo em uma função de minimização, adota-se como função de ampacidade, $f_{2}$, a relação entre ampacidade atual da linha pela ampacidade encontrada para cada cabo:

$$
f_{2}=\frac{I_{\text {atual }}}{I_{\text {nova }}}
$$

onde:

$$
\begin{array}{ll}
I_{\text {atual }}: & \begin{array}{l}
\text { ampacidade da linha na situação atual sem } \\
\text { recapacitação; }
\end{array} \\
I_{\text {nova }}: & \text { ampacidade calculada para o cabo considerado. }
\end{array}
$$

\subsection{Maximização de Possibilidade de ex- pansão}

O objetivo desta função é mensurar qual a sobra de corrente e consequentemente, qual a temperatura limite de operação da linha de transmissão em função da altura cabo solo.

A fim de normalizar esse valor e transformá-lo em uma função de minimização, $f_{3}$ é dada pela relação da ampacidade do cabo atual pela ampacidade calculada considerando a nova temperatura de projeto.

$$
f_{3}=\frac{I_{\text {atual }}}{I_{\text {calculada }}}
$$

onde:

$$
\begin{array}{ll}
I_{\text {atual }}: & \begin{array}{l}
\text { ampacidade da linha na situação atual sem } \\
\text { recapacitação; } \\
\text { ampacidade calculada considerando a sobra } \\
\text { de alculadara cabo solo. }
\end{array}
\end{array}
$$

Para o cálculo da ampacidade excedente, são seguidos os seguintes passos:

\section{Passo a: Identificação do ponto de menor altura cabo solo e cálculo da nova flecha.}

Identificar para cada cabo em análise, qual o ponto cuja altura cabo solo se aproxima mais da altura mínima permitida. Neste ponto considera-se então a altura cabo solo como sendo a altura mínima e recalcula-se o valor da flecha.

$$
h_{\text {cabosolonova }}=\left(f_{\text {nova }}+h_{a}\right)-h_{\text {violao }}
$$

onde: 


$\begin{array}{ll}h_{\text {cabosolonova }}: & \text { valor da altura cabo solo igualada a altura } \\ & \text { mínima; } \\ f_{\text {nova }}: & \text { novo valor de flecha que é calculado para } \\ & \text { a nova altura cabo solo; } \\ h_{a}: & \text { altura de fixação do cabo na estrutura } \\ & \text { anterior; } \\ h_{\text {violao }}: & \text { valor da altitude do ponto de violação. }\end{array}$

\section{Passo b: Cálculo do novo valor de tração}

Com o valor da nova flecha, calcula-se o novo valor de tração a qual será submetido o cabo condutor com a seguinte equação (Labegalini et al., 1992). Considerando: $C_{1}=\frac{T_{0}}{p}$

$$
f=\frac{T_{0}}{p} \cdot\left(\cosh \left(\frac{A}{2 *\left(C_{1}\right)}\right)-1\right)
$$

onde:

$$
\begin{aligned}
& f_{\text {nova }}: \quad \text { novo valor de flecha calculado para a nova } \\
& \text { altura cabo solo; } \\
& x_{\text {violao }} \text { : distância da estrutura anterior até o ponto onde } \\
& \text { a altura cabo solo é menor (ponto crítico); } \\
& x_{\text {vrtice }} \text { distância da estrutura anterior até o vértice da } \\
& \text { catenária; } \\
& T_{\text {Onova }}: \quad \text { novo valor de tração que será calculado para a } \\
& \text { nova flecha; } \\
& C_{1 \text { novo }} \text { divisão da nova tração pelo peso próprio do } \\
& \text { cabo. } C_{1 \text { novo }}=\frac{T_{0 \text { nova }}}{p} \text {. }
\end{aligned}
$$

\section{Passo c: Cálculo do novo valor de temperatura de projeto}

De posse do novo valor da tração, calcula-se a nova temperatura de projeto com a seguinte equação (Labegalini et al., 1992):

$$
\begin{aligned}
& T_{0 \text { nova }}^{3}+T_{0 \text { nova }}^{2} *\left[\frac{e . s \cdot p^{2} \cdot A}{24 \cdot T_{01}^{2}}+\right. \\
& \text { e.s. } \left.\alpha .\left(t_{\text {projnova }}-t_{1}\right)-T_{01}\right]-\frac{e . s \cdot p^{2} \cdot A^{2}}{24}=0
\end{aligned}
$$

onde:
Tonova:
valor da tração na temperatura calculada considerando a nova flecha;
$T_{01}$ : $\quad$ valor da tração na temperatura da condição regente;
$t_{1}$ : temperatura na condição regente;
$t_{\text {projnova }}:$ nova temperatura de projeto que se deseja calcular;
e: $\quad$ módulo de elasticidade do condutor;
$s: \quad$ área de seção transversal do condutor;
$\rho: \quad$ peso unitário do condutor;
A: $\quad$ comprimento do vão regulador;
$\alpha: \quad$ coeficiente de dilatação térmica linear do condutor.

\section{Passo d: Cálculo do novo valor da ampacidade}

Depois de calculado o valor da nova temperatura de projeto, calcula-se a nova ampacidade da linha de transmissão para então calcular o acréscimo relativo à situação vigente. A nova ampacidade se calcula conforme (Labegalini et al., 1992):

$$
I=\sqrt{\frac{\left(q_{r}+q_{c}-q_{s}\right) \cdot 10^{3}}{r}}
$$

onde:

$$
\begin{array}{ll}
q_{s}: & \begin{array}{l}
\text { do ganho de calor por radiação solar } \\
\end{array} \\
& \left(q_{s}=204 . d\right) ; \\
d: & \text { diâmetro nominal do cabo }[\mathrm{m}] ; \\
q_{c}: & \text { perda de calor do cabo; } \\
q_{r}: & \text { perda de calor por irradiação; } \\
r: & \text { resistência do condutor à temperatura de } \\
& \text { equilíbrio. }
\end{array}
$$

O valor da temperatura de projeto influencia no cálculo de qc e qr que são determinados da seguinte forma (Labegalini et al., 1992):

$$
\begin{array}{r}
q_{\text {cnova }}=945,6 \cdot\left(t_{\text {projnova }}-t_{0}\right) \cdot 10^{-4} \\
\cdot\left[0,32+0,43 .(45946,8 \cdot d . V)^{0,52}\right]
\end{array}
$$

onde:

$$
\begin{array}{ll}
q_{\text {cnova }}: & \text { perda de calor do cabo; } \\
V: & \text { velocidade do vento; } \\
t_{\text {projnova }}: & \text { nova temperatura de projeto calculada; } \\
t_{0}: & \text { temperatura do meio ambiente }\left[{ }^{\circ} \mathrm{C}\right] ; \\
d: & \text { diâmetro nominal do cabo }[\mathrm{m}] .
\end{array}
$$




$$
q_{\text {rnova }}=179,2 \cdot 10^{3} . \varepsilon . d .\left[\left(\frac{T_{\text {projnova }}}{1000}\right)^{4}-\left(\frac{T_{0}}{1000}\right)^{4}\right]
$$

onde:

$$
\begin{array}{ll}
q_{\text {rnova }}: & \text { perda de calor por irradiação. }[\mathrm{W} / \mathrm{m}] ; \\
\varepsilon: & \begin{array}{l}
\text { emissividade }- \text { para cabos de alumínio é } \\
\text { recomendado } \epsilon \approx 0,5 ;
\end{array} \\
d: & \begin{array}{l}
\text { diâmetro nominal do cabo }[\mathrm{m}] ; \\
T_{\text {projnova }}: \\
\text { temperatura absoluta de projeto nova }(273+ \\
\left.t_{\text {projnova }}\right)[\mathrm{K}] ;
\end{array} \\
& \text { temperatura absoluta do ambiente }\left(273+t_{0}\right) .
\end{array}
$$

Desta forma, calcula-se a nova ampacidade pela equação (28), já que o valor de qs permanece o mesmo $\left(q_{s}=\right.$ 204.d) e a resistência é um dado do cabo.

$$
I_{\text {nova }}=\sqrt{\frac{\left(q_{\text {rnova }}+q_{\text {cnova }}-q_{s}\right) \cdot 10^{3}}{r}}
$$

\subsection{Minimização de custo de instalação}

O custo de instalação dos cabos para cálculo da minimização é calculado em função do peso de cabo utilizado e seu comprimento.

O cálculo do custo de implantação de novas estruturas não será considerado, pois esta possibilidade foi descartada ao se desconsiderar os cabos cuja altura cabo solo não atinjam o valor da altura mínima. A função de minimização do custo de instalação $f_{4}$ é calculada dividindo-se o custo do cabo que está sendo analisado pelo maior custo dos cabos considerados multiplicado por 1,2. O fator de multiplicação é utilizado para eliminar a possibilidade de um valor maior que $1 \mathrm{em} f_{4}$.

$$
f_{4}=\frac{\text { custo }_{\text {cabo }}}{\text { custo }_{m x} \cdot 1,2}
$$

onde:

$$
\begin{aligned}
& \text { custo }_{\text {cabo }} \text { : custo do cabo que está sendo analisado; } \\
& \text { custo }_{m x} \text { : custo do cabo mais caro. }
\end{aligned}
$$

O custo do cabo é calculado multiplicando-se o peso do cabo (pesototal) em $\mathrm{kg}$ pelo preço do cabo (preço) em $\mathrm{R} \$ / \mathrm{kg}$ conforme equação a seguir:

$$
\text { custocabo }=\text { pesototal } * \text { preo }
$$

O preço do cabo é fornecido pelo fabricante e o peso total é calculado da seguinte forma:

$$
\text { pesototal }=n * L * p
$$

onde:

$n$ : número de cabos da linha de transmissão (circuito simples são 3 e circuito duplo são 6);

$L: \quad$ comprimento total de cabo por fase;

$p$ : $\quad$ peso unitário do cabo.

O cálculo do comprimento dos cabos pode ser feito pela equação da catenária (Labegalini et al., 1992):

$$
L=\sqrt{h^{2}+4 \cdot\left(\frac{T_{0}}{p}\right)^{2} \cdot \operatorname{senh}^{2}\left(\frac{A}{2 \cdot \frac{T_{0}}{p}}\right)}
$$

onde:

$L: \quad$ comprimento do cabo pela equação da catenária para vãos desnivelados;

$T_{0}$ : tração do cabo;

$A$ : comprimento do vão;

$h$ : diferença de altura entre as estruturas.

Após concluído o cálculo do custo para cada cabo, utiliza-se o valor máximo, que será usado como custo $_{m x}$, e comparase com os outros valores encontrados conforme equação (29).

\subsection{Minimização de perda}

Em Nascimento et al. (2009), é mostrado um modelo determinístico para a determinação das perdas em função do valor determinístico da temperatura e da corrente definida no projeto da linha de transmissão.

$$
P_{J}=n_{f} \cdot R_{\text {cond }} \cdot\left(F_{C} \cdot I\right)^{2}
$$

onde:

$$
\begin{array}{ll}
P_{J}: & \text { perda determinística por efeito Joule; } \\
n_{f}: & \text { número de fases; } \\
R_{c o n d}: & \text { resistência do condutor em } \Omega / \mathrm{km} ; \\
F_{C}: & \text { fator de carga; } \\
I: & \text { corrente elétrica nominal. }
\end{array}
$$

O cálculo da perda por efeito Joule é feita para a corrente na temperatura de projeto da linha de transmissão e para o valor 
da nova ampacidade calculada em função da sobra de altura cabo solo.

A função de minimização do custo de instalação $f_{5}$ é calculada dividindo-se a perda calculada na temperatura de projeto pela perda calculada para a nova ampacidade.

$$
f_{5}=\frac{\text { perd } d a_{\text {proj }}}{\text { perda } a_{\text {maxproj }}}
$$

onde:

$$
\begin{array}{ll}
\text { perda } a_{\text {proj }}: & \text { perda calculada pela corrente na } \\
& \text { temperatura de projeto; } \\
\text { perda } a_{\text {maxproj }}: & \text { máxima perda calculada pela corrente na } \\
& \text { temperatura de projeto. }
\end{array}
$$

\subsection{Minimização de esforço em estrutu- ras}

Os esforços em estruturas podem ser longitudinais, que é função da tração de ruptura dos cabos, transversais, que é função do diâmetro do cabo e os esforços verticais, função do peso do cabo.

Os esforços verticais têm pouco impacto na estrutura, no entanto os esforços longitudinais e os esforços transversais têm mais impacto na determinação das estruturas e, portanto a função de minimização $f_{6}$ tem por objetivo considerar este acréscimo nos esforços das estruturas quando da determinação do cabo.

A função de minimização $f_{6}$ é calculada somando- se: a relação da força transversal que o cabo que está sendo simulado provoca na estrutura pela maior força transversal encontra da dentre os cabos simulados mais a relação da força longitudinal que o cabo que está sendo simulado provoca na estrutura pela maior força longitudinal encontrada dentre os cabos simulados.

$$
f_{6}=\frac{F_{v c a b o}}{F_{v m x}}+\frac{T_{c a b o}}{T_{\max }}
$$

onde:

$F_{\text {vcabo }}: \quad$ força que o cabo que está sendo simulado provoca nos pontos de suspensão;

$F_{v m x}: \quad$ maior valor de força provocada nos pontos de suspensão dentre os cabos simulados;

$T_{\text {cabo: }} \quad$ tração do cabo que está sendo simulado;

$T_{\max }$ : maior valor de tração encontrado entre os cabos simulados.
$O$ valor da força $F v$ é calculado por:

$$
F_{v}=\left[\frac{a_{i}+a_{j}}{2}\right] \cdot f_{v}
$$

ou seja,

$$
F_{v}=a_{m} \cdot f_{v}
$$

onde:

$f_{v}: \quad$ força resultante da pressão de vento;

$a_{m}$ : vão médio na estrutura.

E finalmente $f_{v}$ pode ser calculado por:

$$
f_{v}=q_{0} \cdot d
$$

onde:

$f_{v}: \quad$ força resultante da pressão de vento;

$d$ : diâmetro do cabo, em m;

$V_{p}$ : velocidade de vento de projeto, $[\mathrm{m} / \mathrm{s}]$;

$\rho: \quad$ massa específica do ar;

$q_{0}: \quad$ pressão de vento, em N/m² igual a

$$
q_{0}=\frac{1}{2} \rho \cdot V_{p}^{2}
$$

Substituindo (39) em (38):

$$
f_{v}=\frac{1}{2} \rho \cdot V_{p}^{2} \cdot d
$$

A massa específica do ar é calculada por:

$$
\rho=\frac{1,293}{1+0,00367 . t}\left[\frac{16000+64 . t-A L T}{16000+64 . t+A L T}\right]
$$

onde:

$t: \quad$ temperatura coincidente, em ${ }^{\circ} \mathrm{C}$;

$A L T: \quad$ altitude média da implantação da linha, em $\mathrm{m}$. 


\subsection{Problema de Otimização}

Os valores calculados para as funções objetivos estão normalizados, ou seja, apesar de se tratarem de valores com ordens de grandeza diferentes, relacionou-se cada critério por um valor máximo especificado, a fim de torná-los dentro do intervalo zero e um.

A fim de se enfatizar quais os critérios mais relevantes para o projetista da recapacitação, foram adotados também diferentes pesos, de modo a efetuar a soma de todas as funções objetivo e encontrar um valor que possa ser comparado com os outros cabos. O objetivo deste método é possibilitar uma comparação com o critério da otimalidade de Pareto para embasar as conclusões. A equação da função objetivo é (Coello, 1999):

$$
F O=w_{1} \cdot f_{1}+w_{2} \cdot f_{2}+w_{3} \cdot f_{3}+w_{4} \cdot f_{4}+w_{5} \cdot f_{5}+w_{6} \cdot f_{6}
$$

Antes de se calcular os valores de $F O$ para cada cabo disponível, e assim escolher aquele com o menor valor, estabeleceuse um critério de atributos assinalados para cada solução e critério a fim de possibilitar uma comparação das alternativas e composição do conjunto Pareto-Ótimo sobre os quais se decide sobre o melhor cabo (menor $F O$ ).

Constatou-se a necessidade de estabelecimento desses atributos, pois para as linhas estudadas, os cabos disponíveis apresentaram soluções indiferentes entre si, ou seja, cada um dos cabos apresentaram vantagens e desvantagens com relação aos outros.

Para tanto, propõe-se um método de comparação no qual se estabelecem atributos para cada solução analisada, os quais podem assumir valores 0 ou 1 . Assim, se para um determinado critério, se a solução é não dominada, atribui-se o valor 1 e se ela é dominada, atribui-se valor 0 . No final das simulações somam-se cada um esses atributos por cabo. Esta soma representa o desempenho geral do cabo em comparação com os outros. Em seguida estabelece-se uma linha de corte para que sejam descartados os cabos com pior desempenho, ou que poucas vezes chegaram a soluções consideradas não dominadas. As soluções restantes são então as soluções Paretoótimas.

Sobre esse conjunto de soluções, se dá a escolha definitiva do cabo pelo Método do Critério Global Ponderado, ou seja, pelo menor da função objetivo obtida pela ponderação dos critérios de avaliação.

A Figura 2 apresenta um fluxograma geral da metodologia proposta.

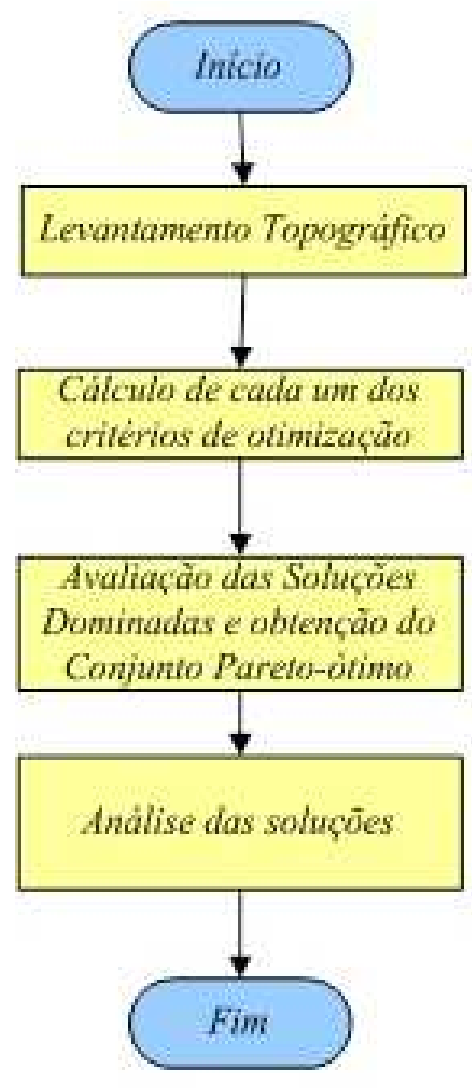

Figura 2: Fluxograma da metodologia geral.

\section{RESULTADOS}

Nesta seção, são apresentados os resultados obtidos a partir da metodologia proposta, supondo a necessidade de aumento de temperatura de uma linha de transmissão $230 \mathrm{kV}$ Figueira - Apucarana de $75^{\circ} \mathrm{C}$ para $90^{\circ} \mathrm{C}$ no estado do Paraná.

O primeiro passo foi selecionar os cabos (disponíveis no mercado) mais apropriados a serem utilizados para recapacitação da linha em análise.

Em linhas de $230 \mathrm{kV}$ são usados em geral os cabos 636 kCM chamado GROSBEAK ou o cabo 795 kCM denominado DRAKE. As estruturas metálicas destas linhas têm suas árvores de carregamento apropriadas para estes dois cabos. Portanto, para evitar a necessidade de cálculo estrutural das torres, foram selecionados para a simulação, cabos com características mecânicas de peso, tração e diâmetro parecidas com os cabos mencionados, os quais estão apresentados na Tabela 3. 
Tabela 3: Tipos de cabos utilizados.

\begin{tabular}{ccccccc}
\hline Cabo & Bitola kCM & Nome do Cabo & Tração de Ruptura $(\mathrm{kgf})$ & Área $\left(\mathrm{mm}^{2}\right)$ & Diâmetro $(\mathrm{mm})$ & Peso $(\mathrm{kgf} / \mathrm{m})$ \\
\hline 1 & 636,0 & GROSBEAK & 11440 & 374,79 & 25,16 & 1,3029 \\
2 & 795,0 & DRAKE & 14300 & 468,51 & 28,13 & 1,6286 \\
3 & 740,8 & FLINT & 11041 & 375,4 & 25,16 & 1,0299 \\
4 & 927,2 & GREELEY & 13821 & 469,8 & 28,15 & 1,2892 \\
5 & 700,0 & $18 / 19$ & 8111 & 355 & 24,45 & 0,9775 \\
6 & 900,0 & $24 / 13$ & 9221 & 456 & 27,73 & 1,2561 \\
7 & 636,0 & T-GROSBEAK & 11427 & 374,8 & 25,16 & 1,3028 \\
8 & 795,0 & T-DRAKE & 14286 & 468,5 & 28,13 & 1,6286 \\
9 & 733,0 & AMSTERDAM & 12522 & 418,5 & 23,5 & 1,113 \\
10 & 839,0 & BRUSSELS & 13878 & 477,2 & 25,14 & 1,275 \\
11 & 922,0 & STOCKHOLM & 15958 & 527,5 & 26,4 & 1,406 \\
12 & $636 \mathrm{~S}$ & GROSBEAK ACSS & 9432 & 374,6 & 25,15 & 1,301 \\
13 & $795 \mathrm{~S}$ & DRAKE ACSS & 11747 & 468,6 & 28,14 & 1,627 \\
14 & $630 \mathrm{C}$ & GROSBEAK ACCR & 11612 & 385 & 25,5 & 1,101 \\
15 & $795 \mathrm{C}$ & DRAKE ACCR & 14606 & 484 & 28,6 & 1,384 \\
16 & $636 \mathrm{TW}$ & OSWEGO & 11728 & 390 & 23,6 & 1,111 \\
17 & $795 T W$ & WABASH & 13592 & 449 & 25,2 & 1,28 \\
\hline
\end{tabular}

Como não se admite qualquer tipo de violação de altura cabo solo, os cabos 1, 2, 5, 6, 7, 8, 12 e 13 foram já prontamente descartados, ou seja, cabos que não causam qualquer tipo de violação de altura cabo solo são: $3,4,9,10,11,14,15,16$ e 17.

Após descartados os cabos cuja solução seja inviável, é feita a comparação de cada parâmetro entre os cabos para se estabelecer as soluções dominadas e não dominadas. Para tanto, a Tabela 4 apresenta os valores de cada critério de otimização para cada cabo restante.

Foi estipulado o atributo 1 para as soluções não dominadas e 0 para as soluções dominadas; considerada uma tolerância de 5\% no comparativo entre os parâmetros para que fossem consideradas não dominadas soluções próximas da solução ótima; e então a somado os valores destes pesos de comparação por cabo para ter um parâmetro de comparação entre os mesmos. Os valores dos somatórios dos atributos para comparação de cada critério de otimização para esses cabos estão apresentados na Tabela 5.

Como todos os cabos remanescentes apresentam soluções não dominadas, elas passam então a compor o conjunto Pareto-Ótimo.

A fim de se escolher a melhor solução do conjunto ParetoÓtimo, passa-se a seguir, a uma análise da função objetivo
Tabela 4: Parâmetros de comparação entre os cabos por critério.

\begin{tabular}{|c|c|c|c|c|c|c|}
\hline \multirow{2}{*}{ Cabo } & \multicolumn{6}{|c|}{ Parâmetros de comparação entre os cabos } \\
\cline { 2 - 7 } & $f_{1}$ & $f_{2}$ & $f_{3}$ & $f_{4}$ & $f_{5}$ & $f_{6}$ \\
\hline 3 & 0,0972 & 0,4447 & 0,3039 & 0,0155 & 0,4436 & 0,6851 \\
\hline 4 & 0,0972 & 0,3856 & 0,2633 & 0,0194 & 0,4741 & 0,8066 \\
\hline 9 & 0,0700 & 0,4259 & 0,2741 & 0,0503 & 0,4262 & 0,7003 \\
\hline 10 & 0,0776 & 0,3911 & 0,2200 & 0,0576 & 0,4434 & 0,7623 \\
\hline 11 & 0,0683 & 0,3680 & 0,2362 & 0,0635 & 0,4564 & 0,8383 \\
\hline 14 & 0,0771 & 0,4306 & 0,2319 & 0,0663 & 0,4472 & 0,7059 \\
\hline 15 & 0,0770 & 0,3712 & 0,1991 & 0,0833 & 0,4786 & 0,8349 \\
\hline 16 & 0,0769 & 0,4368 & 0,2350 & 0,0669 & 0,4273 & 0,6801 \\
\hline 17 & 0,0754 & 0,3996 & 0,2515 & 0,0771 & 0,444 & 0,7554 \\
\hline
\end{tabular}

para diversos valores de pesos associados a cada critério de otimização, segundo a Tabela 6 .

Assim, segundo os pesos da Tabela 6:

- o caso 1 não prioriza nenhum critério de otimização;

- o caso 2 prioriza a minimização do custo; 
Tabela 5: Atributos para comparação dos critérios de otimização.

\begin{tabular}{|c|c|c|c|c|c|c|}
\hline \multirow{2}{*}{ Cabo } & \multicolumn{6}{|c|}{ Parâmetros de comparação entre os cabos } \\
\cline { 2 - 7 } & $f_{1}$ & $f_{2}$ & $f_{3}$ & $f_{4}$ & $f_{5}$ & $f_{6}$ \\
\hline 3 & 1 & 3 & 0 & 8 & 8 & 8 \\
\hline 4 & 1 & 8 & 3 & 7 & 2 & 2 \\
\hline 9 & 8 & 3 & 2 & 6 & 8 & 8 \\
\hline 10 & 6 & 6 & 7 & 5 & 8 & 4 \\
\hline 11 & 8 & 8 & 6 & 4 & 6 & 2 \\
\hline 14 & 6 & 3 & 6 & 4 & 8 & 8 \\
\hline 15 & 6 & 8 & 8 & 0 & 2 & 2 \\
\hline 16 & 6 & 3 & 6 & 3 & 8 & 8 \\
\hline 17 & 6 & 6 & 3 & 1 & 8 & 4 \\
\hline
\end{tabular}

Tabela 6: Pesos para critérios de otimização.

\begin{tabular}{|c|c|c|c|c|c|c|}
\hline \multirow{2}{*}{ Casos } & \multicolumn{7}{|c|}{ Pesos } \\
\cline { 2 - 7 } & $w_{1}$ & $w_{2}$ & $w_{3}$ & $w_{4}$ & $w_{5}$ & $w_{6}$ \\
\hline 1 & 1 & 1 & 1 & 1 & 1 & 1 \\
\hline 2 & 1 & 1 & 1 & 6 & 1 & 1 \\
\hline 3 & 1 & 1 & 1 & 1 & 1,5 & 2,6 \\
\hline 4 & 1 & 1 & 1,5 & 1 & 1 & 2,6 \\
\hline
\end{tabular}

- o caso 3 prioriza a minimização das perdas;

- o caso 4 prioriza a maximização da ampacidade excedente.

\section{Caso 1: Simulação sem Priorizar Nenhum Critério}

A Tabela 7 apresenta os valores de FO e o somatório dos atributos para cada critério de otimização.

Numa primeira análise, excluem-se os cabos que produziram piores soluções. Assim, estabelecendo uma linha de corte em 34, descartam-se os cabos 3, 4, 15 e 17, restando os cabos 9, $10,11,14$ e 16 .

A seguir, é necessário pesquisar se a série de estruturas da linha em questão é para que tipo de bitola. Como para essa linha, o projeto da série é para cabo 636, e os cabos de alguns cabos remanescentes têm bitolas maiores (733, $922 \mathrm{MCM})$ é necessária uma análise mais detalhada dos cabos:

- os cabos 14 e 16 podem ser instalados, sendo que o cabo 16 (636TW denominado $O S W E G O$ ) obteve o menor valor de FO, sendo escolhido como melhor solução.
- os cabos 9 e 10, que têm respectivamente o segundo e terceiro menor valor de $F O$ dentre os cabos remanescentes, possuem um valor de tração maior que o cabo 636 o requer uma análise detalhada da possibilidade de utilizar um valor menor de tração na EDS para não haver impacto na força longitudinal nas estruturas.

- o cabo 11 necessita de estudo mais detalhado pois além da tração, seu peso e diâmetro são maiores que o cabo utilizado no projeto da torre e pode causar esforços indesejáveis na estrutura. O valor de $F O$ para este cabo é o maior dentre os remanescentes, portanto já não seria selecionado.

\section{Caso 2: Simulação Priorizando Custo}

Em geral a tomada de decisão quanto a qual solução adotar não é simplesmente técnica, sendo o custo um fator fundamental nesta decisão.

A segunda simulação considerou um peso na função de minimização do preço para "penalizar" o cabo mais caro. Na comparação dos preços o cabo mais caro é considerado 6 vezes o preço do mais barato e este valor é usado como peso $w_{4}$.

A Tabela 8 apresenta os valores de $F O$ e do somatório dos atributos para esse caso.

A soma dos atributos considerando apenas a função de minimização $f_{4}$, que se refere ao custo, é 8 (valor máximo) apenas no cabo 3, ou seja, no critério custo o cabo FLINT é uma solução não dominada e portanto a melhor solução.

Considerando os valores de $F O$, percebe-se que o cabo 3 (740,8 - FLINT) também é a melhor soluação. Este cabo possui todos os parâmetros para determinação dos esforços menores ou iguais ao cabo 636, portanto pode ser aplicado.

\section{Caso 3: Simulação Priorizando Perda}

A minimização das perdas deve ser considerada simultaneamente com a minimização dos esforços pois cabos com maior diâmetro possuem menores perdas. Se os esforços não forem considerados, a solução pode ficar mascarada. Assim, serão considerados pesos para as funções de minimização das perdas e de minimização dos esforços. Os pesos considerados foram 1,5 para as perdas $\left(w_{5}\right)$ que corresponde à maior perda dividida pela menor e 2,6 para os esforços $\left(w_{6}\right)$, que é o maior esforço dividido pelo menor.

A Tabela 9 apresenta os valores de $F O$ e do somatório dos atributos para esse caso. 
Tabela 7: Tipos de cabos utilizados.

\begin{tabular}{ccccc}
\hline Cabo & Bitola MCM & Nome do Cabo & Valor de $F O$ & Somatório dos Atributos \\
\hline 3 & 740,8 & FLINT & 1,9900 & 28 \\
4 & 927,2 & GREELEY & 2,0462 & 23 \\
9 & 733,0 & AMSTERDAM & 1,9467 & 35 \\
10 & 839,0 & BRUSSELS & 1,9520 & 36 \\
11 & 922,0 & STOCKHOLM & 2,0306 & 34 \\
14 & 630C & GROSBEAK ACCR & 1,9589 & 35 \\
15 & $795 \mathrm{C}$ & DRAKE ACCR & 2,0440 & 26 \\
16 & 636TW & OSWEGO & 1,9230 & 34 \\
17 & $795 \mathrm{TW}$ & WABASH & 2,0030 & 28 \\
\hline
\end{tabular}

Tabela 8: Resultados que priorizam custo - Caso 2.

\begin{tabular}{ccccc}
\hline $\mathrm{N}^{\circ}$ do Cabo & Bitola MCM & Nome do Cabo & Valor de $F O$ & Somatório dos Atributos \\
\hline 3 & 740,8 & FLINT & 2,0675 & 8 \\
4 & 927,2 & GREELEY & 2,1433 & 7 \\
9 & 733,0 & AMSTERDAM & 2,1979 & 6 \\
10 & 839,0 & BRUSSELS & 2,2399 & 5 \\
11 & 922,0 & STOCKHOLM & 2,3480 & 4 \\
14 & $630 \mathrm{C}$ & GROSBEAK ACCR & 2,2904 & 4 \\
15 & $795 \mathrm{C}$ & DRAKE ACCR & 2,4607 & 0 \\
16 & $636 \mathrm{TW}$ & OSWEGO & 2,2575 & 3 \\
17 & $795 \mathrm{TW}$ & WABASH & 2,3884 & 1 \\
\hline
\end{tabular}

Tabela 9: Resultados que priorizam perdas e esforços nas estruturas - Caso 3.

\begin{tabular}{ccccccc}
\hline \multirow{2}{*}{$\mathrm{N}^{\circ}$ do Cabo } & \multirow{2}{*}{ Bitola MCM } & \multirow{2}{*}{ Nome do Cabo } & \multirow{2}{*}{ Valor de FO } & \multicolumn{4}{c}{ Somatório dos Atributos } \\
\cline { 5 - 7 } & & & $f_{5}$ & $f_{6}$ & soma \\
\hline 3 & 740,8 & FLINT & 3,3080 & 8 & 8 & 16 \\
4 & 927,2 & GREELEY & 3,5739 & 2 & 2 & 4 \\
9 & 733,0 & AMSTERDAM & 3,2802 & 8 & 8 & 16 \\
10 & 839,0 & BRUSSELS & 3,3935 & 8 & 4 & 12 \\
11 & 922,0 & STOCKHOLM & 3,6001 & 6 & 2 & 8 \\
14 & $630 \mathrm{C}$ & GROSBEAK ACCR & 3,3120 & 8 & 8 & 16 \\
15 & $795 \mathrm{C}$ & DRAKE ACCR & 3,6192 & 2 & 2 & 4 \\
16 & $636 \mathrm{TW}$ & OSWEGO & 3,2248 & 8 & 8 & 16 \\
17 & $795 \mathrm{TW}$ & WABASH & 3,4337 & 8 & 4 & 12 \\
\hline
\end{tabular}

A soma dos atributos considerando apenas as funções de minimização $f_{5}$ e $f_{6}$ que se referem respectivamente à perda e ao aumento dos esforços é 16 (valor máximo) para os cabos 3, 9, 14 e 16 constituindo-se um conjunto Pareto-ótimo.
Considerando os valores de $F O$, percebe-se que desta vez o cabo 16 (636TW - OSWEGO) passa a ser a solução ótima. Este cabo possui todos os parâmetros para determinação dos esforços próximos do cabo 636, portanto pode ser aplicado. 


\section{Caso 4: Simulação Priorizando Ampacidade Exce- dente}

A maximização da ampacidade excedente também deve ser considerada simultaneamente com a minimização dos esforços, pois o cálculo da ampacidade leva em conta o diâmetro do cabo e o aumento do mesmo leva a maiores esforços. Se os esforços não forem considerados, a solução ótima pode produzir valores indesejáveis de esforços. Assim serão considerados pesos para as funções de maximização da ampacidade excedente e de minimização dos esforços. Os pesos considerados foram 1,5 para a ampacidade excedente $\left(w_{3}\right)$ que corresponde à maior ampacidade excedente dividida pela menor e 2,6 para os esforços $\left(w_{6}\right)$, que é o maior esforço dividido pelo menor.

A Tabela 10 apresenta os valores de $F O$ e do somatório dos atributos para esse caso.

A soma dos atributos considerando apenas as funções de minimização $f_{3}$ e $f_{6}$ que se referem respectivamente à ampacidade excedente e ao aumento dos esforços, não chega a 16 (valor máximo) em nenhum dos cabos, ou seja, não há solução totalmente não dominada. Os maiores valores encontrados foram nos cabos 14 e 16, que serão analisados pela função objetivo.

Considerando os valores de $F O$, percebe-se que cabo 16 (636TW - OSWEGO) é a melhor solução. Este cabo possui todos os parâmetros para determinação dos esforços próximos do cabo 636.

Analisando-se os resultados, pode-se concluir que a escolha do cabo restringiu-se a dois tipos ( 3 e 16). Fazendo uma comparação específica entre as novas temperaturas aproximadas de operação dos esses dois cabos (Tabela 11), percebe-se que o cabo OSWEGO (tipo 16) constitui uma solução mais cara porém produz uma considerável folga para novas recapacitações.

Tabela 11: Comparação das novas temperaturas de operação.

\begin{tabular}{|c|c|c|}
\hline $\begin{array}{c}\text { Parâmetro } \\
\text { Relativo }\end{array}$ & $\begin{array}{c}\text { Cabo 3 - 740,8 } \\
\text { FLINT }\end{array}$ & $\begin{array}{c}\text { Cabo 16 - } \\
\text { 636TW } \\
\text { OSWEGO }\end{array}$ \\
\hline $\begin{array}{c}\text { Máxima } \\
\text { temperatura } \\
\text { aproximada de } \\
\text { operação }\end{array}$ & $95^{\circ} \mathrm{C}$ & $130^{\circ} \mathrm{C}$ \\
\hline
\end{tabular}

A escolha propriamente dita entre os dois melhores cabos para cada linha tem a ver com a política da empresa em se priorizar ou não o custo de implantação em detrimento do custo de futuras recapacitações.

\section{CONCLUSÃO}

Esse artigo propôs uma metodologia multicritério para seleção de cabos em projetos de recapacitação de linhas de transmissão.

Diferentemente do que é feito nas empresas do setor, que testam de 1 a 2 possibilidades, a metodologia faz uma busca exaustiva, ou seja, automatiza o processo de cálculo das grandezas físicas incluindo todas as opções de cabos disponíveis. Nesse processo, calculam-se ampacidade, custo, flecha, nova temperatura de projeto e perdas entre os diversos tipos de cabos existentes no mercado. Em seguida, estes dados subsidiam a formulação de um problema de otimização multiobjetivo, que realiza a escolha do melhor cabo a ser utilizado em função do parâmetro que se deseja enfatizar. Ou seja, pondera adequadamente os objetivos envolvidos no processo de escolha de um cabo. Os critérios utilizados neste trabalho foram maximização de altura cabo-solo, maximização da ampacidade, maximização da sobrevida da linha em função da ampacidade excedente, minimização dos custos de implantação, perdas e acréscimo de esforços nas estruturas.

O problema de otimização envolvido foi resolvido pelo Método do Critério Global Ponderado, que se mostrou eficaz para estabelecer comparação entre os diversos parâmetros e objetivos. Ele permitiu que, através de uma função objetivo composta pelas várias funções de otimização, se faça uma comparação simultânea das possibilidades, ganhandose qualidade na análise.

No entanto, uma dificuldade encontrada foi que não existe uma solução totalmente não dominada ou totalmente dominada. Devido à quantidade de parâmetros de comparação e à quantidade de cabos, todas as alternativas tem algumas soluções não dominadas. A solução encontrada foi então a de estabelecer um peso para soluções dominadas e outro para soluções não dominadas a fim de definir uma linha de corte e diminuir o campo de comparação.

Os resultados obtidos foram satisfatórios e se mostraram coerentes ratificando a metodologia como uma ferramenta eficaz no balizamento de novos projetos de recapacitação.

Essa metodologia permite a análise de todos os cabos possíveis de serem utilizados em um projeto de recapacitação (e não apenas alguns selecionados pela experiência do projetista), sistematiza a obtenção da solução possibilitando a avaliação não só de aspectos tipicamente utilizados (ampacidade, altura cabo-solo, custos, perdas e esforços) mas, agrega também o aspecto relacionado a sobrevida de linhas, ou seja, 
Tabela 10: Resultados que priorizam ampacidade excedente e esforços nas estruturas - Caso 4.

\begin{tabular}{ccccccc}
\hline \multirow{2}{*}{$\mathrm{N}^{\circ}$ do Cabo } & \multirow{2}{*}{ Bitola MCM } & \multirow{2}{*}{ Nome do Cabo } & \multirow{2}{*}{ Valor de FO } & \multicolumn{4}{c}{ Somatório dos Atributos } \\
\cline { 5 - 7 } & & & $f_{5}$ & $f_{6}$ & soma \\
\hline 3 & 740,8 & FLINT & 3,2381 & 0 & 8 & 8 \\
4 & 927,2 & GREELEY & 3,4685 & 3 & 2 & 5 \\
9 & 733,0 & AMSTERDAM & 3,2041 & 2 & 8 & 10 \\
10 & 839,0 & BRUSSELS & 3,2818 & 7 & 4 & 11 \\
11 & 922,0 & STOCKHOLM & 3,4900 & 6 & 2 & 8 \\
14 & $630 \mathrm{C}$ & GROSBEAK ACCR & 3,2043 & 6 & 8 & 14 \\
15 & $795 \mathrm{C}$ & DRAKE ACCR & 3,4795 & 8 & 2 & 10 \\
16 & $636 \mathrm{TW}$ & OSWEGO & 3,1287 & 6 & 8 & 14 \\
17 & $795 \mathrm{TW}$ & WABASH & 3,3374 & 3 & 4 & 7 \\
\hline
\end{tabular}

vislumbra uma solução que admita a utilização do cabo por mais tempo.

\section{REFERÊNCIAS}

ABNT (1985). NBR 5422/1985. projeto de linhas aéreas de transmissão de energia elétrica., Technical report, Associação Brasileira de Normas Técnicas, Rio de Janeiro.

Coello, C. (1999). An updated survey of evolutionary multiobjective optimization techniques: state of the art and future trends, Proceedings of the 1999 Congress on Evolutionary Computation, 1999. CEC 99., Vol. 1.

Dutra, J. F., Lago, F. F. and Cesar, E. L. (2005). Recapacitação de lt's $230 \mathrm{kv}$ com novos cabos termorresistentes, XVII Seminário Nacional de Produção e Transmissão de Energia Elétrica, Curitiba, PR.

Hoffmann, J. N. and Fonseca, H. E. (2003). Custo de estruturas metálicas em projetos de linhas de transmissão, em função do cabo condutor, temperatura de projeto e perfil do terreno, XVII Seminário Nacional de Produção e Transmissão de Energia Elétrica, Uberlândia, MG.
Labegalini, P. R., Labegalini, J. A., Fuchs, R. D. and Almeida, M. T. (1992). Projetos Mecânicos das Linhas Aéreas de Transmissão, 2a. edn, Edgard Blücher.

Nascimento, C. A. M., Guimarães, M. F., Motta, I. L. M. and Filho, E. B. G. (2009). A experiência da CEMIG no uso de condutores especiais nas fases de viabilidade, projeto e construção de linhas aéreas de transmissão., $S e$ minário Nacional de Produção e Transmissão de Energia Elétrica, Recife, PE.

Oliveira, C. M. F. (2000). Recapacitação de linhas de transmissão, Master's thesis, Pontifícia Universidade Católica do Rio de Janeiro, Rio de Janeiro.

Stephan, J. C. S. and Costa, F. C. (2008). Recapacitação e cálculo da capacidade de carga de torres metálicas de linhas de transmissão, 3o. Congresso Latino Americano de Construção Metálica, São Paulo, SP.

Wiedmer, R. S., Souza JR, O. H., Silva, V. and Hoffmann, J. N. (2007). Recapacitação de linhas de transmissão $138 \mathrm{kv}$ utilizando cabo de alumínio liga 6201, XIX Seminário Nacional de Produção e Transmissão de Energia Elétrica, Rio de Janeiro, RJ. 